\title{
Desarrollo de habilidades de estudio de los alumnos tutorados de la licenciatura en gerontología
}

Development of skills of tutored students in the Bachelor's degree study in

gerontology

Desenvolvimento de competências de estudo de estudantes tutelado grau em

gerontologia

Ana Rosa Can Valle

Facultad de Enfermería, Universidad Autónoma de Campeche, México

anarocan@uacam.mx

Betty Sarabia Alcocer

Facultad de Medicina, Universidad Autónoma de Campeche, México

bmsarabi@uacam.mx

\section{Resumen}

En el ámbito educativo, el saber estudiar y el que los estudiantes apliquen una metodología de estudio, han sido una constante preocupación. El docente debe motivar en el estudiante el interés de la enseñanza de estrategias, para potencializar, controlar y regular sus procesos mentales de aprendizaje, lo cual permitirá mejorar su rendimiento y su capacidad de aprender autónomamente a partir de sus propios recursos. Las habilidades de estudio tienen como intención contribuir en el logro del éxito académico. Objetivo. Identificar la forma de estudiar y prepararse para sus exámenes de los Estudiantes del Nivel Superior.

Método. Enfoque cualitativo, alcance descriptivo, diseño no experimental. Muestra 68 sujetos de estudio de la Licenciatura en Gerontología. Se aplicó un cuestionario ¿Cómo es tu forma de estudiar?, está integrado por cinco dimensiones: Lugar, Planificación, Clase, Estudio, Exámenes, Se utilizó el programa estadístico SPSS, versión 19 para su análisis. Resultados. En la dimensión Lugar, el 69.10 \% cuida que exista una adecuada; Planificación, se encontró que el $7.4 \%$ dedica de 5 a 6 horas de estudio a la semana; Clase, 
dice que el $51.5 \%$ anota solo lo más importante de las explicaciones y no intenta copiar todo lo que dice el profesor; Estudio, dice que el $48.5 \%$ siempre subraya las ideas y los datos de interés; Exámenes, se encontró que el 39.7\% nunca realiza un esquema antes de contestar a cada pregunta. Conclusión. Estas actividades que se le aplica a los alumnos, nos sirve para mejorar las técnicas y sus habilidades de estudio.

Palabras claves: Hábitos de estudio, enseñanza, aprendizaje, estrategias, éxito académico.

\section{Abstract}

In the field of education, knowledge study and which students apply a methodology of study, have been a constant concern. The teacher should encourage student interest of teaching strategies, to promote, control and regulate their mental processes of learning, which will improve your performance and your ability to learn autonomously from its own resources. Study skills are intended to contribute to the achievement of academic success. Objective. Identify how to study and prepare for their examinations of the Students of Higher Level.

Method. Qualitative approach, descriptive scope, non-experimental design. It shows 68 subjects of study for the degree course in Gerontology. A questionnaire was applied, how is your way of studying?, consists of five dimensions: Place, Planning, Class, Study, Exams. The statistical program SPSS, version 19 for analysis was used.

Results. In Place dimension, the $69.10 \%$ cares it is suitable; Planning, found that the $7.4 \%$ dedicated from 5 to 6 hours of study per week; Class, says that the $51.5 \%$ record only the most important explanations and not try to copy everything the teacher says; Study, says that the $48.5 \%$ always emphasizes the ideas and data of interest; Exams, found that the $39.7 \%$ never made a scheme before answering each question. Conclusión. These activities, which applies to students, helps us to improve the techniques and study skills.

Key Words: study habits, teaching, learning, strategies, academic achievement. 


\section{Resumo}

Na educação, o estudo do conhecimento e os alunos a aplicar uma metodologia de estudo, têm sido uma preocupação constante. O professor deve incentivar o interesse dos alunos em estratégias de ensino para potenciar, controlar e regular os processos mentais de aprendizagem, o que irá melhorar o seu desempenho e sua capacidade de aprender de forma independente a partir de seus próprios recursos. habilidades de estudo destinam-se a contribuir para a realização do sucesso académico. Objectivo. Identificar como estudar e se preparar para os exames do estudante de nível superior.

Método. abordagem qualitativa, alcance descritivo, design não-experimental. 68 mostra sujeitos do estudo da Licenciatura em Gerontologia. Place, Planejamento, classe, estudo, testes, SPSS versão 19 foi utilizado para análise:? Um questionário Como é a sua maneira de estudar, é composto por cinco dimensões foi aplicado.

Resultados. Coloque a dimensão, o cuidado 69,10\% que não é adequada; Planejamento, constatou que $7,4 \%$ dedica 5 a 6 horas de estudo por semana; Classe, 51,5\% disseram que a pontuação apenas os mais importantes das explicações e não tentar copiar tudo o que o professor diz; Estudo, 48,5\% disseram que sempre enfatiza as ideias e dados de interesse; Testes descobriram que 39,7\% nunca fazer um esboço antes de responder cada pergunta. Conclusão. Estas actividades serão administradas aos alunos, que serve para melhorar as técnicas e habilidades de estudo.

Palavras-chave: hábitos de estudo, ensino, estratégias de aprendizagem, o sucesso acadêmico.

Fecha recepción: Enero 2016

Fecha aceptación: Junio 2016

\section{Desarrollo}

En el ámbito educativo, el saber estudiar y el que los estudiantes apliquen una metodología de estudio, han sido una constante preocupación. El docente debe motivar en el estudiante el interés de la enseñanza de estrategias, para potencializar, controlar y regular sus procesos 
mentales de aprendizaje, lo cual permitirá mejorar su rendimiento y su capacidad de aprender autónomamente a partir de sus propios recursos. Las habilidades de estudio tienen como intención contribuir en el logro del éxito académico.

Los estudiantes, tienen la idea que estudiar es igual a leer, repetir y memorizar. Para poder adquirir habilidades para el estudio, es que ellos desarrollen sus propias técnicas de aprendizaje y no sólo las técnicas concretas que se enseñan.

Según Monereo (1997). El interés de la enseñanza de estrategias reside en la potencialidad que se le da al sujeto para controlar y regular sus procesos mentales de aprendizaje, lo que le permitirá mejorar su rendimiento y sobre todo su capacidad de aprender autónomamente a partir de sus propios recursos. La finalidad del desarrollo de habilidades de estudio y de aprendizaje escolar es lograr el éxito académico a través del dominio de técnicas y alcanzar recursos e instrumentos por los estudiantes.

\section{Justificación}

Esta investigación tiene como finalidad que el alumno adquiera conocimientos para poder aplicarlo en sus habilidades de estudio, en la actualidad los alumnos tiene dificultades para estudiar, lo que ocasiona la deserción escolar.

El instrumento que se aplica a los estudiantes es muy completo, analiza las cinco dimensión que se mencionan más adelante.

Objetivo Identificar la forma de estudiar y prepararse para sus exámenes de los Estudiantes del Nivel Superior.

\section{Material y Métodos}

Esta investigación tiene un enfoque cualitativo, con alcance descriptivo y un diseño no experimental. Con una muestra de 68 sujetos de estudio de la Licenciatura en Gerontología de la Facultad de Enfermería de la Universidad Autónoma de Campeche. El instrumento denominado: ¿Cómo es tu forma de estudiar?, ¿Qué tal estudiante eres? Este instrumento está integrado por cinco dimensiones: Lugar, integrado por seis ítems; Planificación, integrado por nueve ítems; Clase, integrado por ocho ítems; Estudio, integrado por 
diecisiete ítems; Exámenes, integrado por once ítems; un total de cincuenta reactivos. Se utilizó el programa estadístico SPSS, versión 19 para el análisis de resultados.

\section{Resultados}

En la dimensión uno: Lugar, como lo muestra la gráfica 1, el 69.10 \% cuida que exista una adecuada iluminación al estudiar, en contraste solo el $2.9 \%$ nunca lo cuida.

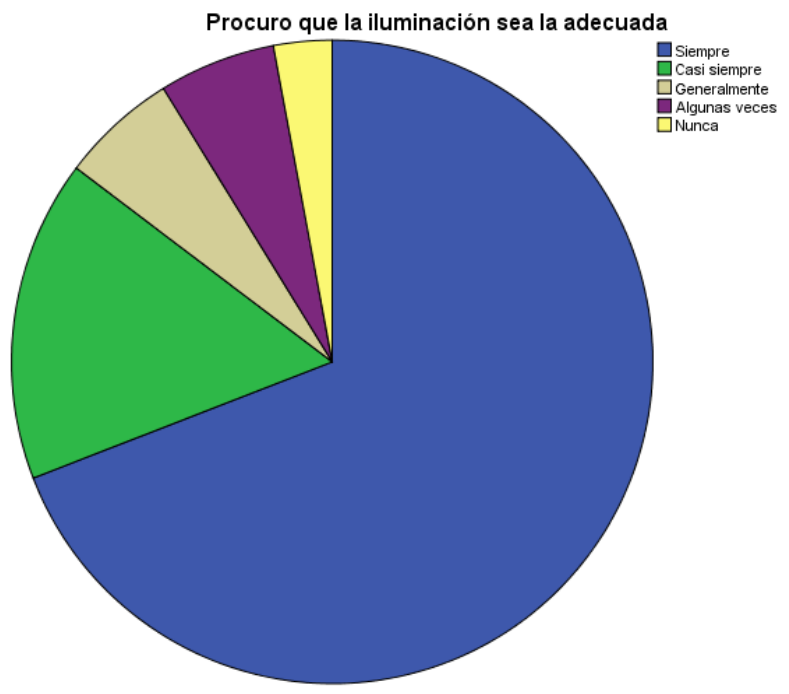

Gráfica 1

El $32.4 \%$ siempre estudia en el mismo lugar, en contraste con el $5.9 \%$ nunca estudia en un lugar fijo. Solamente al $33.8 \%$ siempre le interesa que la temperatura ambiental está controlada. Como lo muestra la gráfica 2.

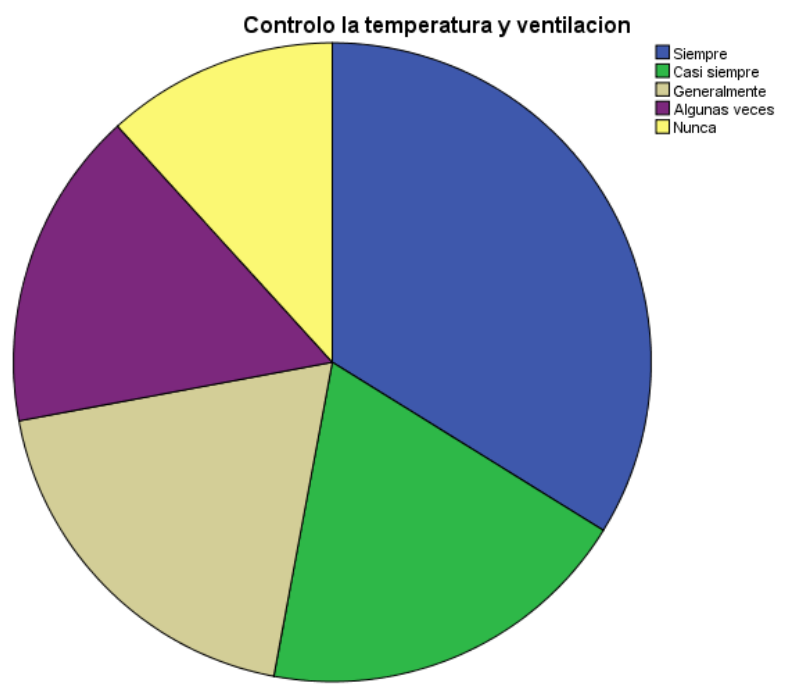

Gráfica 2 
En la dimensión dos: Planificación, se encontró que el 7.4\% dedica de 5 a 6 horas de estudio a la semana, con un día de descanso, en contraste con el $16.2 \%$ que nunca lo hace (gráfica 3).

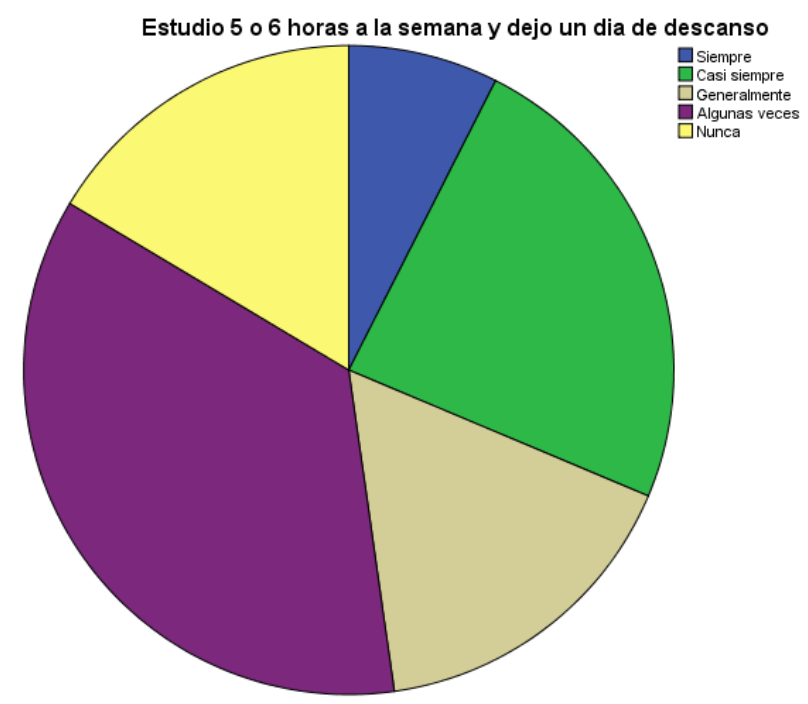

Gráfica 3

El 33.8\% planea su tiempo sin imposición de sus padres (gráfica 4).

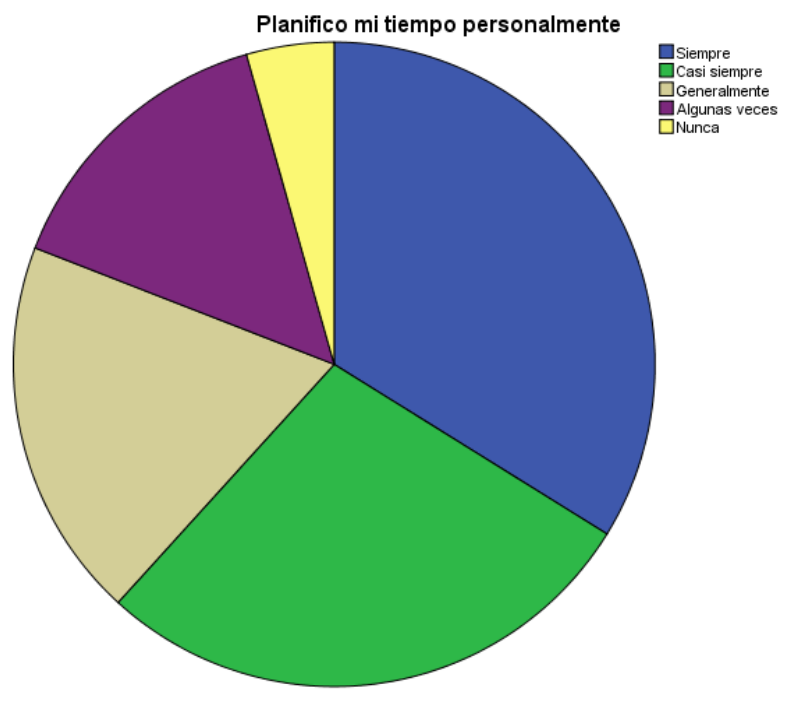

Gráfica 4 
En la dimensión tres: Clase, dice que el 51.5\% anota solo lo más importante de las explicaciones y no intenta copiar todo lo que dice el profesor. Como lo muestra la gráfica 5.

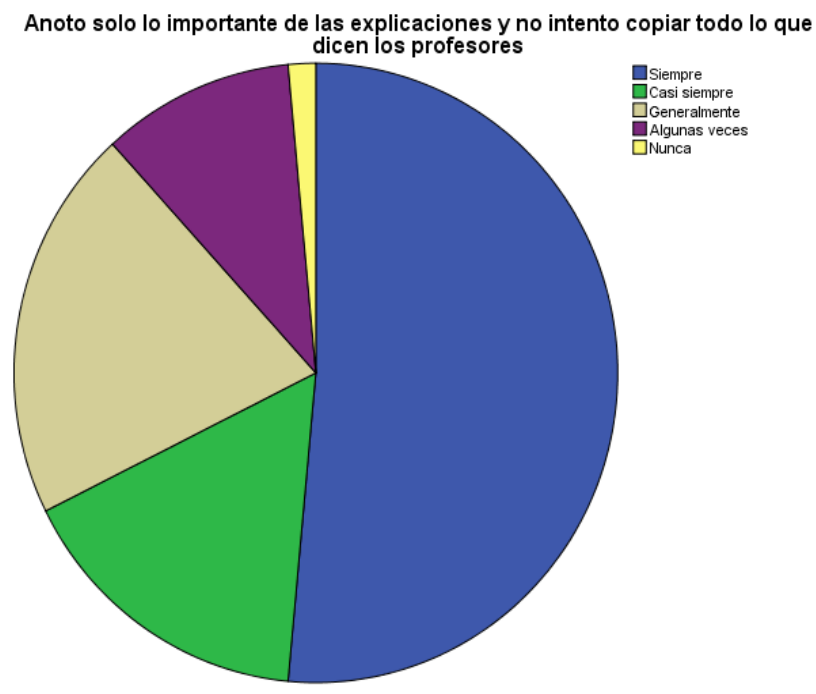

Gráfica 5

El $42.6 \%$ toma nota de ejercicios y explicaciones, y utilizan abreviaturas para ahorrar tiempo al tomar apuntes. El 38.2\% afirma que siempre tiene sus apuntes revisados y ordenados por materia (gráfica 6).

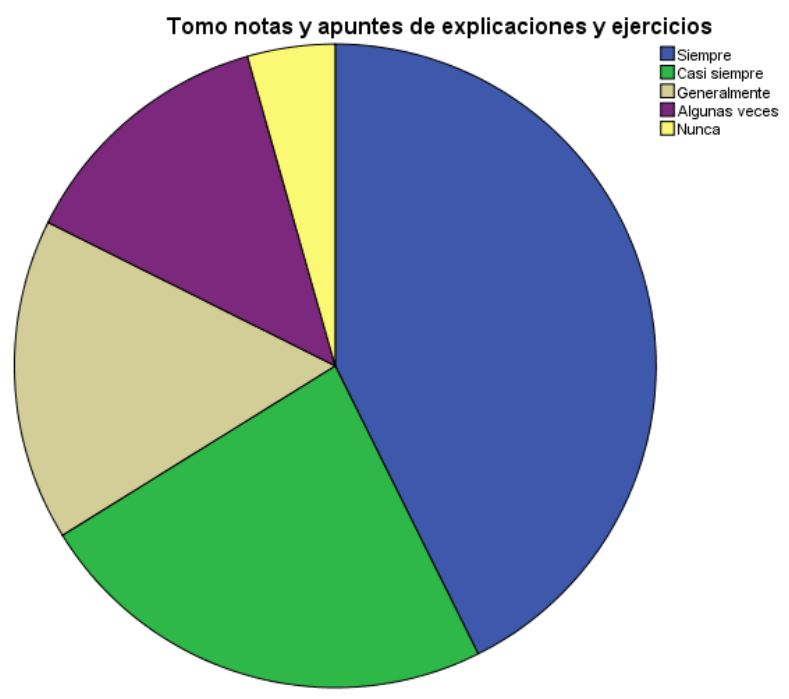

Gráfica 6 
En la dimensión cuatro: Estudio (ver gráfica 7), dice que el 48.5\% siempre subraya las ideas y los datos de interés; el $44.1 \%$ siempre procura que lo subrayado tenga sentido al leerlo.

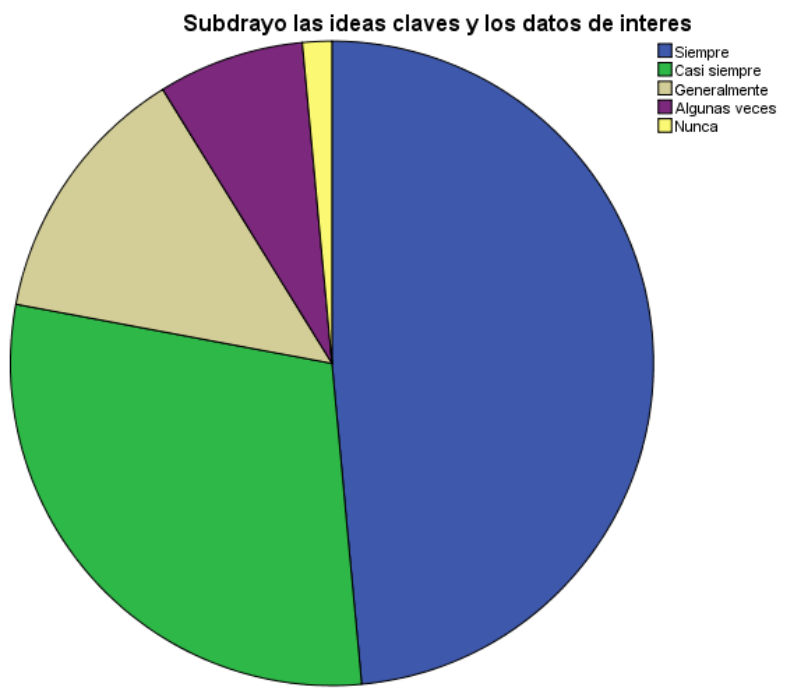

Gráfica 7

Solamente el $20.6 \%$ siempre acude al diccionario cuando tiene duda. Solamente el 5.9\% repasa solo sobre apuntes, esquemas o textos subrayados (gráfico 8).

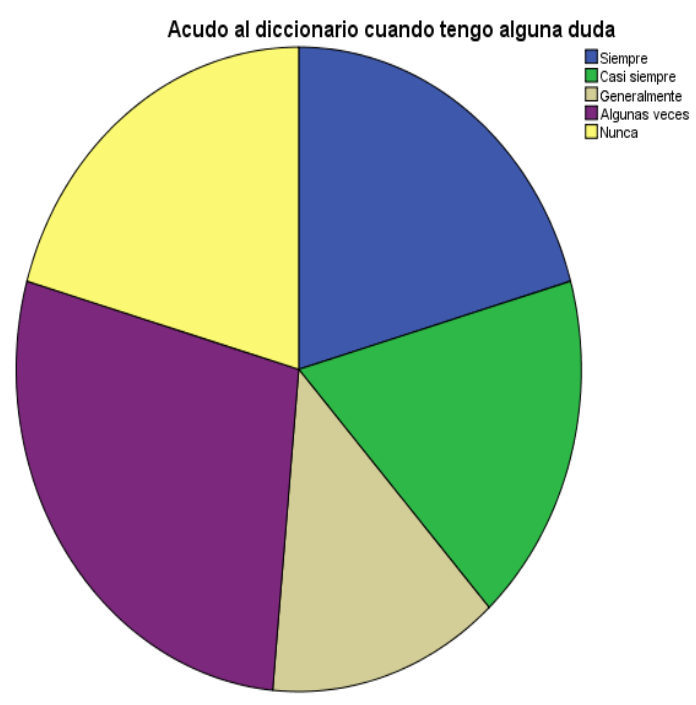

Gráfica 8 
En la dimensión cinco: Exámenes (ver gráfica 9), se encontró que el 39.7\% nunca realiza un esquema antes de contestar a cada pregunta. Solamente el $7.4 \%$ siempre respeta las horas habituales de descanso.

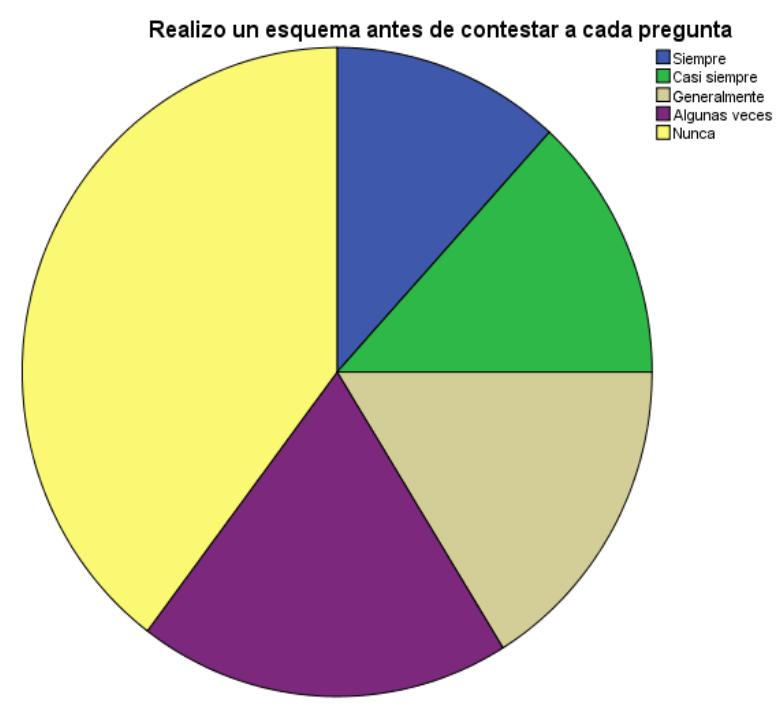

Gráfica 9

El 32.4\% siempre distribuye el tiempo del examen que vaya a dedicar antes de responder.

El 41.2\% siempre confirma la hora, fecha y lugar del examen con suficiente antelación (gráfica 10).

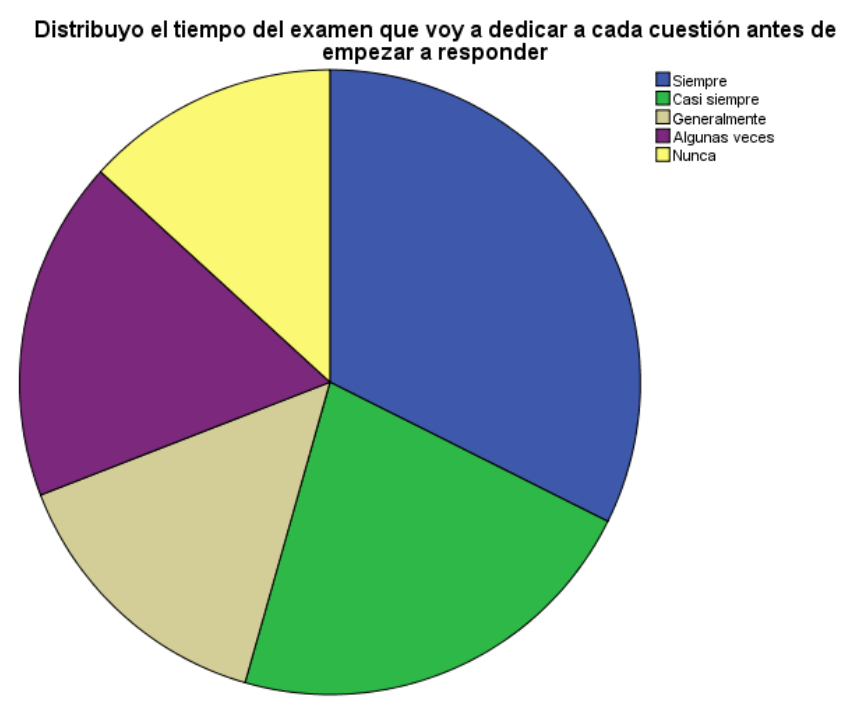

Gráfica 10 


\section{Conclusiones}

En conclusión, la dimensión Lugar, el 69.10 \% cuida que exista una adecuada iluminación al estudiar, esto quiere decir lo importante que es para tener éxito las tareas o el estudio se requiere de esta. En la dimensión Planificación, se encontró que el 7.4\% dedica de 5 a 6 horas de estudio a la semana, con un día de descanso, es importante el tiempo que dedica el alumno al estudio.

En la dimensión Clase, dice que el 51.5\% anota solo lo más importante de las explicaciones y no intenta copiar todo lo que dice el profesor, en algunas ocasiones los alumnos copian lo que dice el profesor.

En la dimensión Estudio, dice que el $48.5 \%$ siempre subraya las ideas y los datos de interés, así al alumno se le hace más fácil a la hora de estudiar y en la Exámenes, se encontró que el 39.7\% nunca realiza un esquema antes de contestar a cada pregunta se encontró que el $39.7 \%$ nunca realiza un esquema antes de contestar a cada pregunta, lo que hicieron el esquema se les facilita a la hora de estudia y poder contestar el examen.

Es importancia hacer mención la importancia de los alumnos sobre las habilidades de estudio para que adquieran un éxito en sus estudios. En la Universidad Autónoma de Campeche, hay una Coordinación del Programa institucional de Tutorías que ofrece cursos y talleres a los tutorados para que adquieran estas habilidades y técnicas a la hora de estudiar. 


\section{Bibliografía}

Álvarez González, M., y otros (1988). Métodos de estudio. Barcelona: Editorial Martínez Roca.

Dela Torre, J. C. (1994). Aprender a pensar y pensar para aprender. Madrid: Centro de Publicaciones del Ministerio de Educación y Ciencia.

Hernández Piña, F. (1990). Aprendiendo a aprender: métodos y técnicas de estudio para alumnos de Educación Primaria y Secundaria. Murcia:

Lerko Print - (1998). Aprendiendo a aprender. Técnicas de estudio. Barcelona: Océano.

Monereo, C. (1990). Enseñara aprender ya pensar en la escuela. Madrid: Aprendizaje.

Monereo, C., Y Castelló, M. (1996). Las estrategias de un aprendizaje Cómo incorporarlas a la práctica educativa. Ed. debé. Barcelona.

Monereo, C., y otros (1996). "Orientación y tutoría educativas en el ámbito de las estrategias de aprendizaje", en R. Bisquerra, Manual de Orientación y Tutoría. Barcelona: Praxis

Ontoria, A., Y otros (1999). Mapas conceptuales. Una técnica para aprender. (S." edición). Madrid: Narcea. 